\title{
Evolution Models of Power Communication Network Management Based on IPv6 Network
}

\author{
Guojun Liu, Fangyuan Li, Nan Wang, Ziwei Hu, Nan Wang, Biyao Huang \\ China Electric Power Research Institute, No.15, Qing He Xiao Ying East Road, Haidian District, Beijing \\ 100192, China
}

\begin{abstract}
IPv6 network management evolution requires the transition from IPv4 / IPv6 dual-stack network management stage to full IPv6 network management. The paper made two models for IPv6 network management based on the requirement of network management and present situation of electric power communication network. One model used IPv4 / IPv6 dual stack power communication network management transition construction method, the other put forward independent network management VPN and network management network to construct full IPv6 network management.
\end{abstract}

KEYWORD: IPv6; Network Management

\section{THE NEXT GENERATION INTERNET}

The next generation of the Internet as a strategic emerging industry in China, the country attaches great importance to it. China has a clear goal in this field: in 2013, small-scale IPv6 commercial pilot will be carried out with a mature business model and technology development route; from 2014 to 2015, large-scale deployment and commercial use will be common, and IPv4 and IPv6 mainstream business can communicate.

Power-related enterprises pay attention to promote IPv6 network applications in the power industry. At present, the power company is still in the pilot IPv6 network construction phase, and the power communications integrated network management system has not yet support IPv6 network management completely, lack of effective transition and evolution path for IPv6 network management.

Most IPv6 network management research focused on IPv6 network topology management [1], IPv6 network management protocol ${ }^{[2]}$, etc., while there is little research in IPv6 integrated network management and IPv6 network management evolutionary path. In this paper, IPv6 network management evolution path was put forward to guide power IPv6 network construction.

\section{EVOLUTION REQUIREMENTS}

\subsection{Network equipment demand}

Currently, the equipment management system of power communications data network basically uses the SNMP protocol. To achieve IPv6 SNMP-based network management, equipment management system of vendors should address the following issues:

- RFC2465, RFC2466, RFC2452, RFC2454 added a lot of IPv6 MIB objects, equipment management system should support these new management projects.

- Network management interface of existing IPv6 network equipment is not uniform; the compatibility problems of equipment from different manufacturers exist.

- For supporting IPv6, network equipment from different vendors has different degree, lack of appropriate standards.

\subsection{Compatible with IPv4 network management}

Upgrading the IP network is a gradual process, IPv4 and IPv6 networks will coexist within a certain period. Therefore, the network management system must support both IPv4 and IPv6 networks management. The system will remain all the functional of IPv4 network management system, and expand related functional for IPv6. Finally, IPv4 and IPv6 management will be integrated into a single system and support for IPv4 MIB and IPv6 MIB 
information collection. Network communication protocols stack can automatically adapt IPv4 or IPv6 network.

\subsection{Support network protocol evolution}

There are large differences among IPv6 network device on the network management standards and specifications. Smooth upgrade requires IPv6 devices being very flexible and easy integration of various equipment manufacturers IPv6 equipment, and adapting to the development of IPv4 and IPv6 network management protocol. In IPv6 transition phase, integrated network management system should support both IPv4 and IPv6 SNMP protocol stack products, such that only through simple configuration can realize the connection network communication protocols IPv4 and IPv6.At the same time, flexible configurable plug-in network interface adapter program should be designed, So that different manufacturers, different models of IPv6 devices can be easily integrated into the unified management of network management systems.

\section{EVOLUTION}

IPv4 network to the pure IPv6 network through the following inevitable three stages: $\mathrm{IPv} 4$ network stage, IPv4 / IPv6 dual-stack IPv6 network stage, and pure IPv6 stage. Electricity communication network management will experience the corresponding development, shown in figure 1.

The first stage: IPv4 network stage. This stage includes communication equipment management system of IPv4 manufacturers, IPv4 professional NMS, IPv4 power communication integrated network management system.

The second stage: the transition phase using dual-stack IPv4 and IPv6 network management system, enabling the management of IPv4 and IPv6 network devices at the same time.

The third stage: full IPv6 network management system phase. Manufacturers of equipment related to the network management system, professional NMS, integrated network management systems are running IPv6 protocol.

\subsection{Transitional phase construction mode}

The mode of IPv6 network management transition phase of construction can use IPv4 network management to bear original service. For new or modified service bearer network, use IPv6 IPv4 / IPv6 dual stack network management. Integrated network management system also supports IPv4 / IPv6 dual stack, as shown in Figure 2.

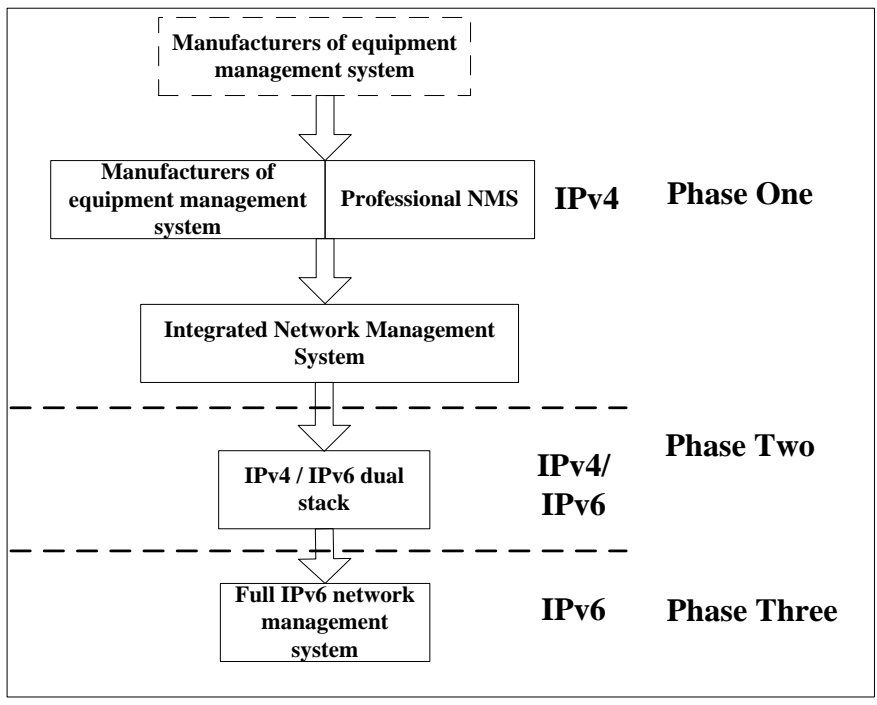

Figure 1. IPv6 network management evolution diagram

In the electric power data communication network, establishing an independent VPN can be used to carry IPv6 Add /Renovation business, the VPN network deployment IPv4 / IPv6 dual-stack equipment or pure IPv6 network management systems. Deployment of integrated network management information in the information network VPN, and make IPv6 VPN network into the integrated network management range.

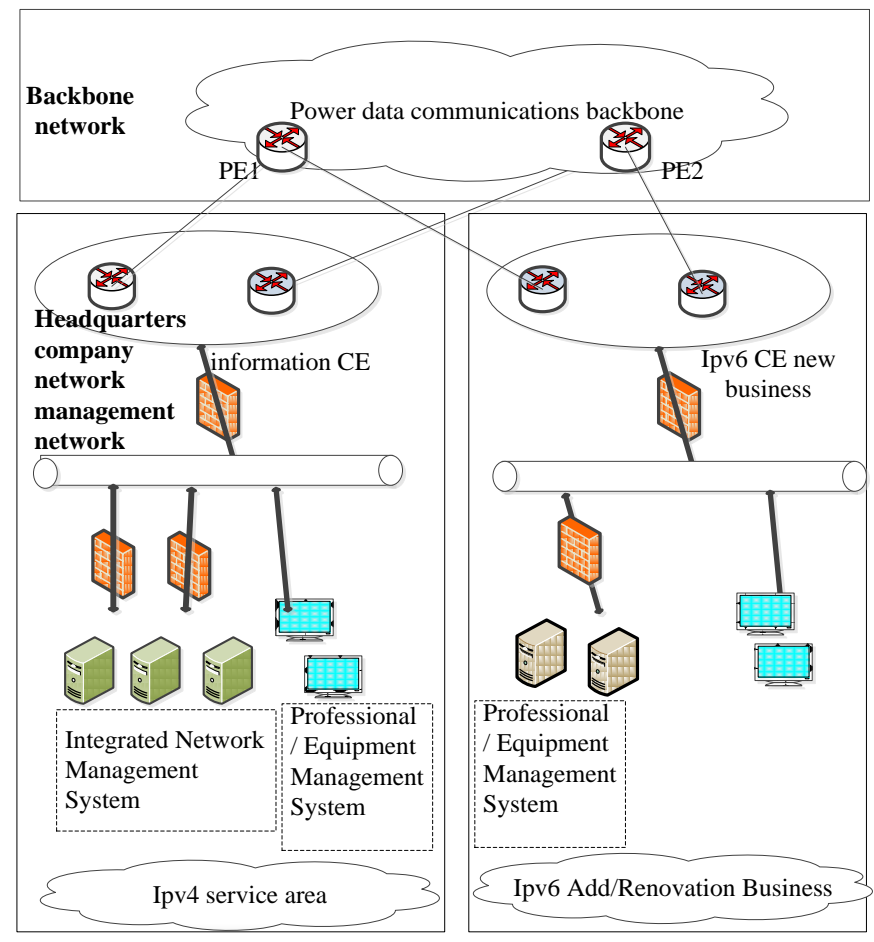

Figure 2. transition phase construction mode

\subsubsection{IPv6 network management system:}

Dual stack network management system should also support for information collection of IPv4 MIB and IPv6 MIB.

Automatic topology discovery can be applied to IPv4/IPv6 dual stack network device. VPN.

Support configuration function of IPv6 MPLS 
The network management system should have automatic or convenient tunnel configuration and management functions, such as automatic discovery or identification of tunnel.

\subsubsection{Northbound Interface}

Alarm Management: In addition to IPv4 generic alarm management, we must also support some certain specific performance alarms of IPv6 devices, such as routers support IPv6 protocol alarm trap / notification outside.

Performance Management: The historical performance data of the device or the number of real-time performance can be reported to Device Management System, SG-TMS can obtain historical performance data of the device or the number of real-time performance on its own initiative.

Traffic management: network interface device should be able to take the initiative to report SG-TMS real-time traffic flow statistics. Support IPv6 traffic management devices include routers and three switches IPv6.

\subsubsection{IPv6 integrated network management function}

Integrated network management data acquisition module should support devices or professional NMS network management system to interface IPv6 north collection of information, including the IPv6 address, IPv6 routing, IPv6 interface information.

Real-time monitoring module supports IPv6 centralized alarm monitoring capabilities, support for IPv6 equipment performance data acquisition, analysis and processing.

Resource management module should support IPv6 device configuration information collection, automatic support for IPv6 network topology discovery, unified identity IPv6 and IPv4 devices equipment, IPv6 tunnel recognition and other functions.

\subsection{Full IPv6 construction mode}

Power data communication network through small-scale pilot IPv6, IPv6 transition phase upgrading will eventually realize IPv6 network covering the power of data communication network, all network systems, and business systems . There are two kinds of models can be used in two all-IPv6 network data communication network system construction.

\subsubsection{Option One: Building Managed VPN}

As shown in Figure 3, in the power data communication backbone network to establish VPN, providing an efficient and safe management information delivery platform for the professional, the manufacturers network management systems.
Managed network includes electricity transmission networks, business networks, support networks and access network. Make the integrated network management and data communications network of professional / equipment management system access to the VPN network. All hosts are IPv6 VPN services, PE, CE, switches and other network equipment and business systems support IPv6 protocol. Meanwhile VPN network must increase strict access control management, allowing only access to their respective network management server IP VPN.

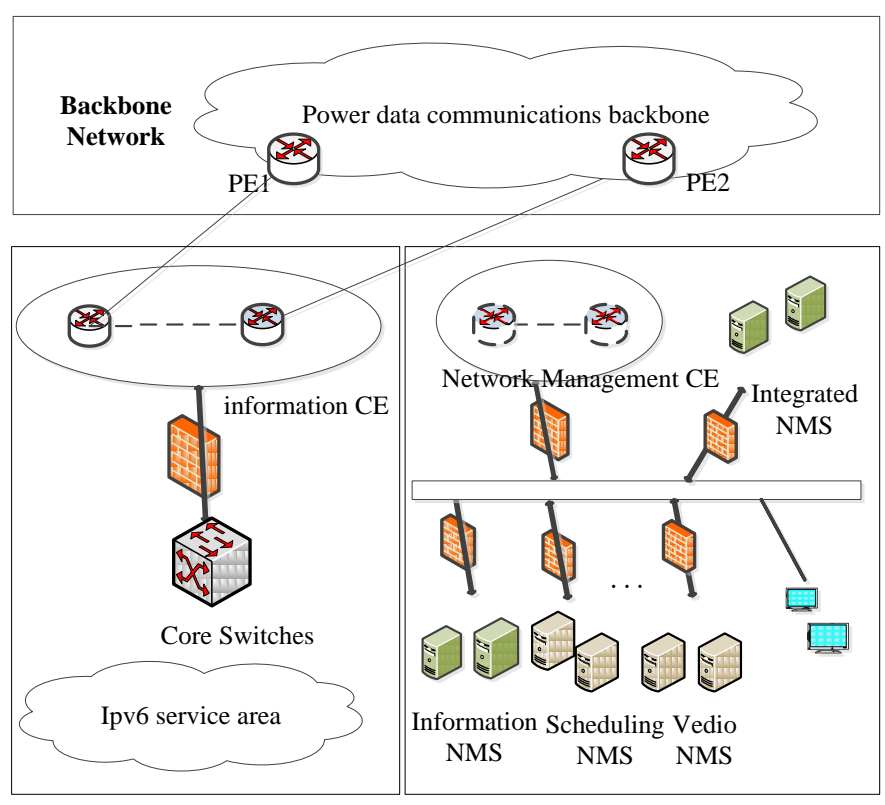

Figure 3. Option One

\subsubsection{Building an independent network management network}

With distribution automation, intelligent building strong, large-scale electric power communication network is growing, professional network or device network management is more and more, so safe and stable operation of the network management system and centralized operation become more difficult. We can establish data network independent with the power of data communication networks, network management scheduling data network, providing a dedicated data communications network for network services, to achieve communication management system, professional / vendor network management and monitoring system for operation of power communication network, monitoring and management specific building program in Figure 4. NMS network is built independent with the power of data communication networks and network scheduling data, the relevant software and hardware companies all the network management system (server, acquisition, FEP, etc.) are deployed in the network management data network, the headquarters responsible for network management network level one, two backbone network communication network management. Provincial companies are responsible 
for level three/four backbone network communication network management.

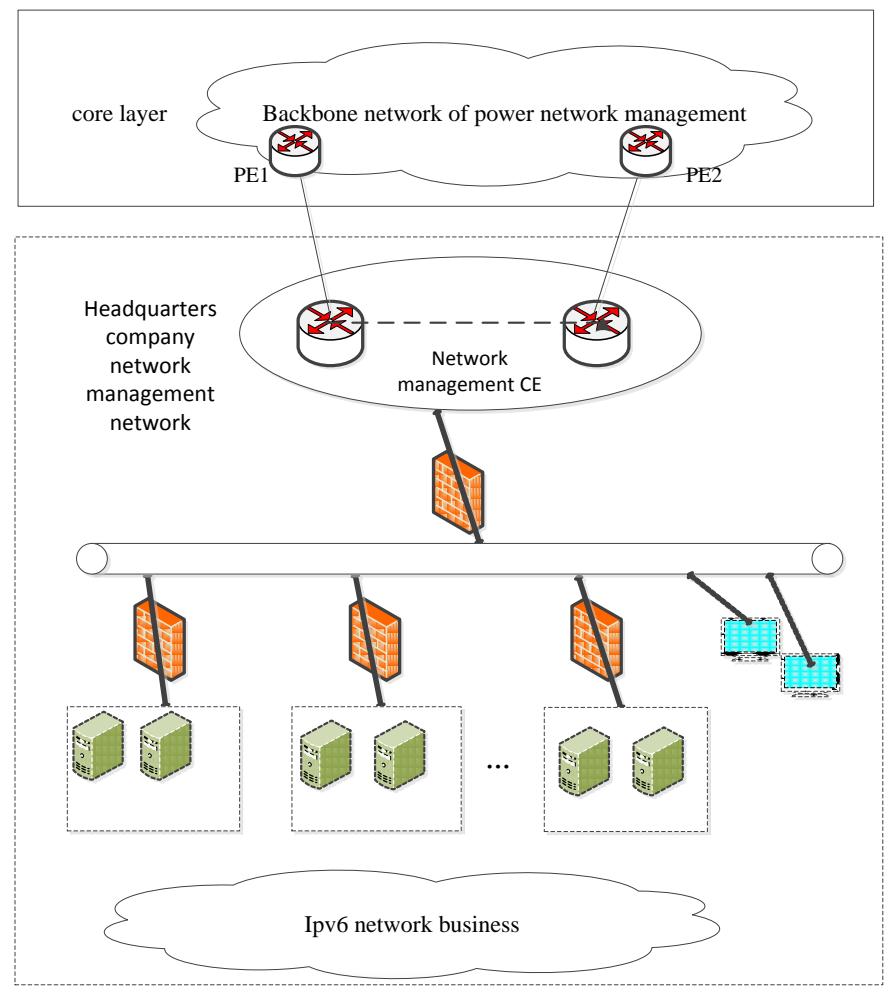

Figure 4. Option Two

\subsubsection{Scheme comparison}

Scheme one is a comprehensive data network management of VPN, the network management system into network management in VPN, and carried on a comprehensive data network. The implementation of the scheme is simple, only need to increase the network management of VPN in data communication in the backbone network, and VPN can support the IPv4 protocol or IPv6 protocol, convenient information network and other business transverse Internet, applicable to IPv6 transition network management. But because the business system integrated data network division in the security area, and the security level is low. The network management system includes many control and configuration commands with the higher requirement on the network VPN security.

Option Two is to build a network management network with data communication network and scheduling data network. The network is relatively comprehensive data network, and security is high; do not take up a comprehensive data network resource. Centralized operation and maintenance and management of the network are convenient. But it needs to reconstruct the network management network based network, and the investment is big.

\section{CONCLUSIONS}

Power communication network based on IPv6 network management development is a gradual process, but also will experience IPv4 network stage, IPv4 / IPv6 dual-stack IPv6 network management network management stage and the whole stage.

Power IPv6 network management transition mode can be used to establish an independent VPN used to carry IPv6 Add / renovation business, the VPN network deployment IPv4 / IPv6 dual-stack equipment management system, integrated information network deployed in VPN, support for dual-stack network equipment administration.

Power full IPv6 network management building can be built in VPN network or independent network management network modes.

\section{REFERENCES}

[1] Zeadally, S. et al.2004. Comparison of End-system IPv6 Protocol Stacks. Communications, IEEE Proceedings-, 151(3):238-242.

[2] Afifi, H. \& Toutain, L.1999. Methods for IPv4-IPv6 transition. Computers and Communications, 1999. Proceedings. IEEE International Symposium on: 478 484. 6 July 1999. Red Sea.

[3] Eun-Young,P.et al.2004.An IPv4-to-IPv6 dual stack transition mechanism supporting transparent connections between IPv6 hosts and IPv4 hosts in integrated IPv6/IPv4 network. Communications, 2004 IEEE International Conference on: 1024-1027.20-24 June 2004.

[4] Miao Xin 2010.Smart distribution grid IPv6 transition technology and strategy. Electric Power Construction, 31(2):1-7.

[5] Heping Hou,et al.2010.Design and implementation of a solution to smooth IPv6 transition. Advanced Intelligence and Awarenss Internet (AIAI 2010), 2010 International Conference on:157-161.23-25 October. Beijing, China. 